\title{
Moderating Effects of Alexithymia on Associations between the Therapeutic Alliance and the Outcome of Brief Psychodynamic- Interpersonal Psychotherapy for Multisomatoform Disorder
}

\section{OPEN ACCESS}

Edited by:

Martina De Zwaan,

Hannover Medical School, Germany

Reviewed by:

Bernhard Strauss,

Friedrich Schiller, Universität Jena,

Germany

Maria Kleinstäuber,

Philipps University of Marburg,

Germany

*Correspondence:

Thomas Probst

thomas.probst@donau-uni.ac.at

these authors have contributed equally to this work and should be

considered co-first authors.

Specialty section:

This article was submitted to Psychosomatic Medicine, a section of the journal Frontiers in Psychiatry

Received: 15 July 2017 Accepted: 14 November 2017 Published: 04 December 2017

Citation:

Probst T, Sattel H, Gündel H, Henningsen P, Kruse J, Schneider G and Lahmann C (2017) Moderating

Effects of Alexithymia

on Associations between the

Therapeutic Alliance and the

Outcome of Brief PsychodynamicInterpersonal Psychotherapy for

Multisomatoform Disorder.

Front. Psychiatry 8:261.

doi: 10.3389/fpsyt.2017.00261

\author{
Thomas Probst ${ }^{1,2 * t}$, Heribert Sattel ${ }^{3 \dagger}$, Harald Gündel ${ }^{4}$, Peter Henningsen ${ }^{3}$, \\ Johannes Kruse ${ }^{5,6}$, Gudrun Schneider ${ }^{7}$ and Claas Lahmann ${ }^{3,8}$
}

${ }^{1}$ Georg-Elias-Müller Institute for Psychology, Georg-August University of Göttingen, Göttingen, Germany, ${ }^{2}$ Department for Psychotherapy and Biopsychosocial Health, Danube University Krems, Krems, Austria, ${ }^{3}$ Department of Psychosomatic Medicine and Psychotherapy, Klinikum rechts der Isar, Technical University of Munich, Munich, Germany, ${ }^{4}$ Department of Psychosomatic Medicine and Psychotherapy, Ulm University, Ulm, Germany, ${ }^{5}$ Department of Psychosomatic Medicine and Psychotherapy, University of Giessen, Giessen, Germany, ${ }^{6}$ Department of Psychosomatic Medicine and Psychotherapy, University of Marburg, Marburg, Germany, ${ }^{7}$ Department of Psychosomatics and Psychotherapy, University Hospital Münster, Münster, Germany, ${ }^{8}$ Department of Psychosomatic Medicine and Psychotherapy, University Medical Center Freiburg, Freiburg, Germany

This secondary analysis of a trial on brief psychodynamic-interpersonal therapy (PIT) for patients with multisomatoform disorder investigated whether alexithymia moderates the associations between the therapeutic alliance and the outcome of PIT and whether moderating effects of alexithymia remain significant when controlling for depression. Eighty-three patients with multisomatoform disorder receiving PIT were statistically analyzed. Moderation analyses were performed with the SPSS macro PROCESS. The primary outcome $(\mathrm{Y})$, self-reported physical quality of life at 9-month after the end of PIT, was measured with the physical component summary (PCS) of the SF-36 Health Survey. The potential moderator $(\mathrm{M})$ alexithymia was operationalized with the Toronto Alexithymia Scale (TAS-20) at pre-treatment and the predictor $(X)$ the therapeutic alliance was rated by both patients and therapists via the Helping Alliance Questionnaire (HAQ) at the end of PIT. Moreover, the PCS at pre-treatment functioned as covariate in all moderation models. When the patients' alliance ratings were analyzed, alexithymia did not moderate associations between the alliance and the outcome. When the therapists' alliance ratings were evaluated, alexithymia moderated the relationship between the alliance and the outcome $(p<0.05)$ : a stronger alliance in the therapists' perspective was beneficial for the outcome only for patients scoring above 61 on the TAS-20. This moderating effect of alexithymia was, however, not statistically significant anymore when adding the pre-treatment depression scores (PHQ-9) as a covariate to the moderation model. The results underline the importance of a good therapists' view of the alliance when treating alexithymic patients and highlight the complex interaction between alexithymia and depression. Future studies are needed to extend the scope of research regarding which psychotherapeutic mechanisms of change are beneficial for which patients.

Keywords: alexithymia, therapeutic alliance, psychodynamic psychotherapy, somatic symptom disorder, outcome 


\section{INTRODUCTION}

Brief psychotherapies are efficacious for patients with multiple medically unexplained physical symptoms [e.g., Ref. (1)]. However, some patients with functional somatic syndromes undergoing psychotherapy also experience negative effects that can be attributed to factors within and outside the context of the psychotherapy (2). Patient variables have been shown to contribute to a more positive or more negative psychotherapy. For example, the short-term outcome of cognitive behavioral therapy (CBT) was more positive for patients suffering from somatoform disorders when they had a psychiatric history, higher psychological symptom severity, less characteristics related to personality-disorder, and a higher mental quality of life (3). Leibbrand et al. (4), however, failed to show associations between the treatment outcome of patients with somatoform disorder and their comorbid anxiety, depression, and personality disorders. In another study, long-term treatment outcomes were more negative for patients with somatization syndrome when the patients had a low acceptance of psychotherapy and less treatment expectations (5). Another patient variable associated with a less favorable outcome of psychodynamic psychotherapy is alexithymia (6). In CBT, alexithymia either did not influence the outcome (7) or had a beneficial effect on the outcome (8). Alexithymia can be described as having difficulties in identifying and describing emotions as well as by an externally oriented thinking style and affect regulation deficits (9-11). Karukivi and Saarijärvi (12) reviewed factors related to the development of alexithymia and identified genetic, environmental, and individual developmental factors. Some studies have shown a correlation between alexithymia and symptoms related to somatization [e.g., Ref. $(11,13)]$. Although this association remained significant even when controlling for depression in the study by Mattila et al. (13), other studies reported that this correlation diminishes when controlling for negative affect such as depression [e.g., Ref. (14-16)].

In psychotherapy, therapists show predominately contempt when working with alexithymic patients (17). Such reactions of the therapists might contribute to the detrimental effect alexithymia exerts on the outcome of some psychotherapies (18). Moreover, alexithymia correlated negatively with the therapeutic alliance, which in turn correlated positively with the outcome in a current study on CBT and interpersonal therapy for patients with depressive disorder (8). A strong therapeutic alliance is associated with a more favorable psychotherapy outcome across all patients (19); yet, some patients benefit more than others from a strong therapeutic alliance. For example, Lorenzo-Luaces et al. (20) found that the therapeutic alliance affected the outcome only in depressed patients with 0-2 prior episodes but not in depressed patients with at least three prior episodes. Zilcha-Mano and Errázuriz (21) reported that symptom severity moderated the alliance-outcome link with more severely distressed patients benefiting more from a strong alliance.

To investigatealexithymia as a moderator of the alliance-outcome relationship, the current study re-analyzed data from a multicenter randomized controlled trial (RCT) on brief psychodynamic-interpersonal therapy (PIT) for patients with multisomatoform disorder
(22). A multisomatoform disorder diagnosis requires at least three current, functionally disabling somatoform symptoms (on at least half of the days over at least 2 years) not sufficiently explained by an organic disease or another mental disorder, and intensive health-care use (23). Although this research question was not initially planned in the context of the RCT, we hypothesized that the therapeutic alliance exerts a more beneficial effect on the outcome of PIT in patients with higher alexithymia than in patients with lower alexithymia. This hypothesis bases on findings that therapists experience more difficulties in the relationship with alexithymic patients $(8,17,18)$ suggesting that it is more important to reduce these difficulties/to establish a strong alliance in alexithymic than non-alexithymic patients. Moreover, we investigated whether alexithymia moderates the relationship between the alliance and the outcome of PIT even when controlling for depression. We had no specific hypothesis here because research on the question whether alexithymia and depression overlap has produced inconsistent results [e.g., Ref. $(24,25)]$.

\section{MATERIALS AND METHODS}

The multicenter randomized controlled trial ["PISO trial" (22)] the data was drawn from was registered (ISRCTN23215121) and approved by the ethics committees of the six participating sites. Informed consent was obtained from all patients.

\section{Study Procedure}

In the PISO trial, $n=107$ patients were randomized to the intervention condition (PIT) and $n=104$ patients were allocated to the control condition (enhanced medical care, EMC). A blocked randomization list (stratified random blocks of four, six, or eight patients) was generated and applied to the sample by the Coordination Centre for Clinical Trials. The follow-up assessment was realized 9-month after the end of the treatment by post.

\section{Patients}

The patients met the diagnostic criteria for multisomatoform disorder (23) and suffered from pain as the predominant symptom. The "Structured Clinical Interview" (SCID) for DSM-IV (26) was used and modified according to the criteria published by Kroenke et al. (23) to diagnose a multisomatoform disorder. Retrospectively, all patients fulfilled the criteria for a somatic symptom disorder according to DSM-5.

\section{Treatment}

The patients of the present study received manualized brief psychodynamic-interpersonal psychotherapy [PIT (27)]. The control condition of the PISO trial (EMC) was not analyzed in the study at hand, since forming a therapeutic alliance was of particular importance in the intervention as compared to the control condition: establishing a therapeutic alliance was an explicit component of the PIT protocol but not of the EMC protocol [see study protocol published as supplement in Ref. (28)].

PIT consisted of 12 weekly individual sessions including the establishment of the therapeutic alliance, the treatment of the somatoform symptoms, their behavioral, emotional, and interpersonal 
correlates, and the discussion of termination issues $(22,27,29)$. Delivery of PIT was controlled for adherence by independent raters (30). Moreover, therapists used checklists to rate their adherence to the PIT manual for each session.

\section{Measures}

The primary outcome was patient-reported physical quality of life at 9-month after the end of PIT (22) and it was operationalized with the Physical Component Summary (PCS) of the SF-36 Health Survey (31). Moreover, patients filled in the Toronto Alexithymia Scale [TAS-20 (9)] at pre-treatment to measure alexithymia. Furthermore, the therapeutic alliance was rated by patients and therapists with the Helping Alliance Questionnaire [HAQ (32)] at the end of PIT; patients but not therapists filled in the HAQ also at 9-month after PIT; therefore, the ratings at the end of PIT were statistically analyzed to have patients' and therapists' alliance ratings measured at the same assessment point. Furthermore, the patients' scores on the depression scale of the Patient Health Questionnaire [PHQ-9 (33)] at pre-treatment were used in the present study to investigate whether the potential moderating effect of alexithymia on the alliance-outcome link is robust even when controlling for depression.

\section{Statistics}

To explore whether alexithymia moderates associations between the patients'/therapists' alliance ratings and the outcome, and whether this is also the case after controlling for depression, moderation models were performed with PROCESS (34). PROCESS is a SPSS macro for moderation and mediation analysis. Within PROCESS, model 1 was selected and the confidence interval was set to $95 \%$. In the moderation models, the alliance ratings (HAQ) at the end of PIT were entered as the predictor (X), physical quality of life (PCS) at 9-month after PIT functioned as the outcome $(\mathrm{Y})$, alexithymia at pre-treatment (TAS-20) was added as the moderator (M), and physical quality of life (PCS) at pre-treatment was entered as covariate (to analyze the PCS change from pre-treatment to 9-month after PIT). In further models, depression (PHQ-9) at pre-treatment was added as second covariate. In case a statistically significant interaction between the predictor the therapeutic alliance (X) and the moderator alexithymia (M) emerged, the JohnsonNeyman Technique was applied to identify the threshold(s) of the moderator $(\mathrm{M})$ where the association between the predictor $(\mathrm{X})$ and the outcome $(\mathrm{Y})$ transition(s) between statistical significance and non-significance (34). All statistical tests were performed two-tailed and the significance value was set to $p<0.05$.

\section{RESULTS}

\section{Sample Description}

Patients' and therapists' HAQ ratings, patients' TAS-20 scores, patients' PHQ-9 scores, as well as patients' PCS scores were available for $N=83$ of all $N=107$ patients receiving PIT. The flow-chart is presented in Figure 1.

These 83 patients (gender: $61.4 \%$ female; age: $\mathrm{M}=48.34$, $\mathrm{SD}=11.27$ ) were treated per protocol by eight therapists and represent the sample of the current study.

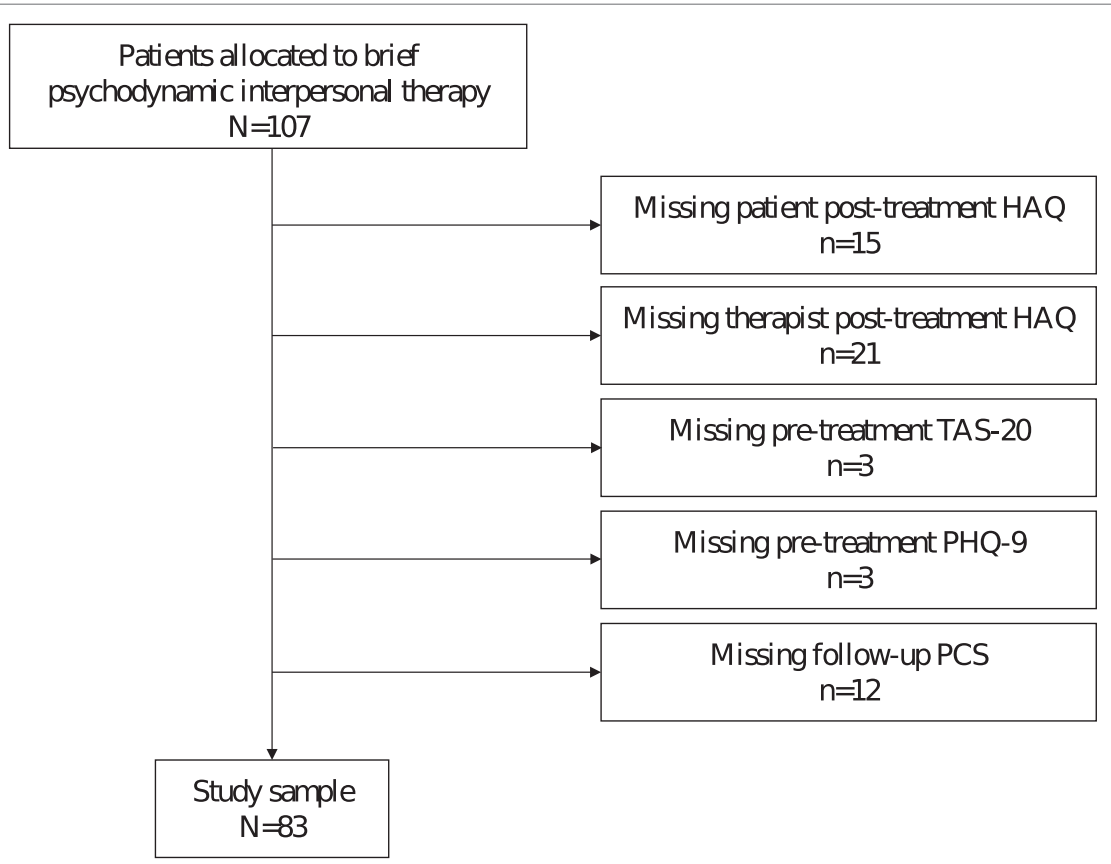

FIGURE 1 | Flow-chart. Note: More missing questionnaires than excluded patients because of multiple missing questionnaires per excluded patient. Abbreviations: HAQ, Helping Alliance Questionnaire; TAS-20, Toronto Alexithymia Scale; PHQ-9, Depression scale of the Patient Health Questionnaire; PCS, Physical Component Summary of the SF-36 Health Survey. 


\section{Correlations between Specific Measures}

The therapists' ratings of their adherence to PIT did not significantly correlate with the TAS-20 scores for any of the 12 sessions ( $r$ ranged from -0.18 to 0.11 ; all $p>0.12$ ). The correlations between the TAS- 20 and the HAQ ratings did not reach statistical significance either (patients' HAQ: $r=-0.08 ; p=0.46$; therapists' HAQ: $r=-0.07 ; p=0.53)$. Yet, the TAS-20 scores were significantly positively related to the PHQ-9 scores $(r=0.41 ; p<0.01)$. The difference scores of the PCS (pre-treatment scores were subtracted from follow-up scores) did not significantly correlate with either the TAS-20 scores $(r=-0.18 ; p=0.10)$ or the HAQ ratings (patients' HAQ: $r=-0.16$; $p=0.15$; therapists' HAQ: $r=0.06 ; p=0.58)$ across all patients.

\section{Alexithymia as a Moderator of the Alliance-Outcome Link without Taking Depression into Account Patients' Alliance}

Table 1 shows that the interaction effect between the patients' alliance ratings and patients' alexithymia on physical quality of life at

TABLE 1 | Results of the moderation analysis investigating alexithymia as a moderator of the association between the patients' alliance ratings and the outcome.

\begin{tabular}{|c|c|c|c|c|c|c|}
\hline \multicolumn{7}{|c|}{ Outcome: PCS at follow-up } \\
\hline $\boldsymbol{R}$ & $R^{2}$ & MSE & $\boldsymbol{F}$ & df1 & df2 & $p$-Value \\
\hline \multirow{2}{*}{0.57} & \multicolumn{6}{|c|}{ Model } \\
\hline & Coefficient & SE & $t$ & $p$-Value & LLCI & ULCI \\
\hline HAQ_P & -3.60 & 3.90 & -0.92 & 0.36 & -11.36 & 4.17 \\
\hline HAQ_P ${ }^{*}$ TAS-20 & 0.05 & 0.08 & 0.58 & 0.57 & -0.12 & 0.21 \\
\hline PCS at pre-treatment & 0.88 & 0.15 & 6.01 & $<0.01$ & 0.59 & 1.17 \\
\hline \multicolumn{7}{|c|}{ Conditional effect of HAQ_P on PCS at follow-up at values of TAS-20 } \\
\hline TAS-20 values & Effect & SE & $t$ & $p$-Value & LLCI & ULCI \\
\hline 10th percentile: 31 & -2.11 & 1.55 & -1.36 & 0.18 & -5.19 & 0.98 \\
\hline
\end{tabular}

PCS, Physical Component Summary of the SF-36 Health Survey; TAS-20, Toronto Alexithymia Scale; HAQ_P. Helping Alliance Questionnaire patient version; LLCI, Iower level of the confidence interval; ULCl, upper level of the confidence interval.

TABLE 2 | Results of the moderation analysis investigating alexithymia as a moderator of the association between the therapists' alliance ratings and the outcome.

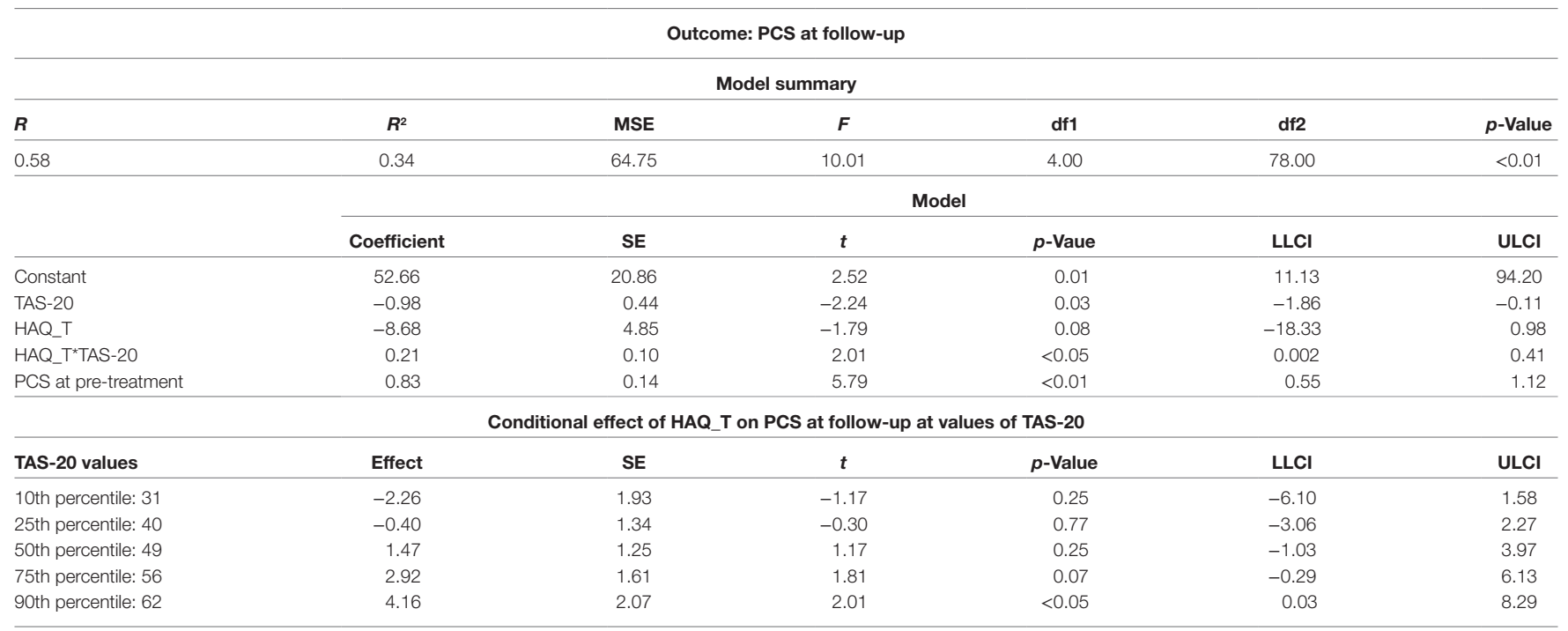

PCS, Physical Component Summary of the SF-36 Health Survey; TAS-20, Toronto Alexithymia Scale; HAQ_T, Helping Alliance Questionnaire therapist version; LLCI, Lower level of the confidence interval; ULCI, Upper level of the confidence interval. 
9-month after PIT did not attain statistical significance $(t=0.58$; $p=0.57)$. Therefore, alexithymia did not moderate the association between the patients' alliance ratings and the outcome.

\section{Therapists' Alliance}

The interaction effect between the therapists' alliance ratings and patients' alexithymia on physical quality of life at 9-month after PIT reached statistical significance $(t=2.01 ; p<0.05$; see
Table 2). This means that alexithymia moderated the association between the therapists' alliance ratings and the outcome. Reported in the lower part of Table 2, a stronger therapists' alliance exerted a significantly beneficial effect on the outcome only at very high values of alexithymia (for the 90th percentile of the TAS-20 scores: $t=2.01 ; p<0.05)$. The moderator value defining the JohnsonNeyman significance region was a TAS-20 score of $61.21(\%$ below: 85.54 ; $\%$ above 14.46 ).

TABLE 3 | Results of the moderation analysis investigating alexithymia as a moderator of the association between the patients' alliance ratings and the outcome when controlling for depression.

\begin{tabular}{|c|c|c|c|c|c|c|}
\hline \multicolumn{7}{|c|}{ Outcome: PCS at follow-up } \\
\hline \multicolumn{7}{|c|}{ Model summary } \\
\hline$R$ & $R^{2}$ & MSE & $\boldsymbol{F}$ & df1 & df2 & $p$-Value \\
\hline \multirow[t]{3}{*}{0.57} & 0.33 & 66.56 & 7.57 & 5.00 & 77.00 & $<0.01$ \\
\hline & \multicolumn{6}{|c|}{ Model } \\
\hline & Coefficient & SE & $t$ & $p$-Value & LLCI & ULCI \\
\hline Constant & 30.37 & 18.48 & 1.64 & 0.10 & -6.42 & 67.17 \\
\hline TAS-20 & -0.24 & 0.40 & -0.60 & 0.55 & -1.05 & 0.56 \\
\hline HAQ_P & -2.92 & 3.98 & -0.74 & 0.46 & -10.84 & 4.99 \\
\hline HAQ_P*TAS-20 & 0.03 & 0.09 & 0.38 & 0.71 & -0.14 & 0.20 \\
\hline PCS at pre-treatment & 0.85 & 0.15 & 5.69 & $<0.01$ & 0.55 & 1.15 \\
\hline PHQ-9 at pre-treatment & -0.17 & 0.18 & -0.91 & 0.37 & -0.53 & 0.20 \\
\hline \multicolumn{7}{|c|}{ Conditional effect of HAQ_P on PCS at follow-up at values of TAS-20 } \\
\hline TAS-20 values & Effect & SE & $t$ & $p$-Value & LLCl & ULCI \\
\hline 10th percentile: 31 & -1.92 & 1.56 & -1.23 & 0.22 & -5.04 & 1.19 \\
\hline 25th percentile: 40 & -1.63 & 1.08 & -1.50 & 0.14 & -3.79 & 0.53 \\
\hline 50th percentile: 49 & -1.34 & 1.04 & -1.29 & 0.20 & -3.41 & 0.73 \\
\hline 75th percentile: 56 & -1.12 & 1.35 & -0.83 & 0.41 & -3.80 & 1.57 \\
\hline 90th percentile: 62 & -0.92 & 1.74 & -0.53 & 0.60 & -4.38 & 2.54 \\
\hline
\end{tabular}

PCS, Physical Component Summary of the SF-36 Health Survey; TAS-20, Toronto Alexithymia Scale; HAQ_P, Helping Alliance Questionnaire patient version; PHQ-9, Depression scale of the Patient Health Questionnaire; LLCI, lower level of the confidence interval; ULCI, upper level of the confidence interval.

TABLE 4 | Results of the moderation analysis investigating alexithymia as a moderator of the association between the therapists' alliance ratings and the outcome when controlling for depression.

\begin{tabular}{|c|c|c|c|c|c|c|}
\hline \multicolumn{7}{|c|}{ Outcome: PCS at follow-up } \\
\hline \multicolumn{7}{|c|}{ Model summary } \\
\hline$R$ & $R^{2}$ & MSE & $\boldsymbol{F}$ & df1 & df2 & $p$-Value \\
\hline \multirow[t]{3}{*}{0.58} & 0.34 & 65.45 & 7.95 & 5.00 & 77.00 & $<0.01$ \\
\hline & \multicolumn{6}{|c|}{ Model } \\
\hline & Coefficient & SE & $t$ & $p$-Value & LLCI & ULCI \\
\hline Constant & 51.28 & 21.25 & 2.41 & 0.02 & 8.97 & 93.59 \\
\hline TAS-20 & -0.92 & 0.47 & -1.98 & 0.05 & -1.85 & 0.01 \\
\hline HAQ_T & -8.21 & 5.01 & -1.64 & 0.11 & -18.18 & 1.76 \\
\hline HAQ_T*TAS-20 & 0.20 & 0.11 & 1.83 & 0.07 & -0.02 & 0.41 \\
\hline PCS at pre-treatment & 0.82 & 0.15 & 5.55 & $<0.01$ & 0.53 & 1.11 \\
\hline PHQ-9 at pre-treatment & -0.08 & 0.18 & -0.41 & 0.68 & -0.44 & 0.29 \\
\hline \multicolumn{7}{|c|}{ Conditional effect of HAQ_T on PCS at follow-up at values of TAS-20 } \\
\hline TAS-20 values & Effect & SE & $t$ & $p$-Value & LLCI & ULCI \\
\hline 10th percentile: 31 & -2.13 & 1.96 & -1.09 & 0.28 & -6.04 & 1.77 \\
\hline 25th percentile: 40 & -0.37 & 1.35 & -0.28 & 0.78 & -3.05 & 2.31 \\
\hline 50th percentile: 49 & 1.39 & 1.27 & 1.09 & 0.28 & -1.14 & 3.93 \\
\hline 75th percentile: 56 & 2.77 & 1.66 & 1.66 & 0.10 & -0.54 & 6.07 \\
\hline 90th percentile: 62 & 3.94 & 2.15 & 1.83 & 0.07 & -0.34 & 8.23 \\
\hline
\end{tabular}

PCS, Physical Component Summary of the SF-36 Health Survey; TAS-20, Toronto Alexithymia Scale; HAQ_P, Helping Alliance Questionnaire patient version; PHQ-9, Depression scale of the Patient Health Questionnaire; LLCl, lower level of the confidence interval; ULCl, upper level of the confidence interval. 


\section{Alexithymia as a Moderator of the Alliance-Outcome Link When Controlling for Depression}

\section{Patients' Alliance}

As summarized in Table 3, alexithymia did also not moderate the association between the patients' alliance at the end of PIT and physical quality of life at 9-month after PIT when adding depression at pre-treatment as a covariate to the moderation model $(t=0.38 ; p=0.71)$.

\section{Therapists' Alliance}

Alexithymia did not significantly moderate the association between therapists' alliance at the end of PIT and physical quality of life at 9-month after PIT anymore when depression at pre-treatment was added as a covariate to the moderation model (see Table 4; $t=1.83 ; p=0.07$ ).

\section{DISCUSSION}

This study re-analyzed data from a multicenter randomized controlled trial on PIT for multisomatoform disorder to investigate whether alexithymia moderates associations between the therapeutic alliance and the outcome of PIT. Across all patients, neither alexithymia [in contrast to Ref. (6)] nor the therapeutic alliance [contrary to Ref. (19)] correlated with the outcome of PIT. However, alexithymia moderated the effect a stronger therapists' alliance exerted on the outcome of PIT: significantly beneficial effects of a stronger therapists' alliance on the outcome emerged only for patients scoring above the TAS-20 threshold of 61 points $(9,35)$ diagnostic for clinically relevant alexithymia. These results fit to other studies highlighting the role of the person of the therapist when working with alexithymic patients $(6$, $17,18)$. However, alexithymia was no significant moderator of the relationship between the therapists' alliance and the outcome anymore when controlling for depression. The non-significant effect of alexithymia when taking depression into account is in line with other studies reporting that symptoms related to somatization did not correlate anymore with alexithymia [e.g., Ref. $(14,16)]$ or associated affect regulation deficits (15) when controlling for negative affect such as depression. Yet, this was not the case in the study by Mattila et al. (13). It has been discussed that alexithymia and depression are overlapping constructs [e.g., Ref. $(24,25)]$ and the correlation between alexithymia and depression became also significant in the current study $(r=0.41)$ fitting to the moderate relationship ( $r=0.43$ ) found in the meta-analysis by Li et al. (36). Another study investigating alexithymia as a moderator between psychotherapeutic mechanisms of change and the outcome did not control for depression but for general symptom distress: Terock et al. (37) found that the patients' psychotherapy motivation (more specifically "degree of suffering") improved the short-term outcome only for patients with lower TAS-20 scores (37). As a stronger alliance in the therapists' view was beneficial for patients with higher TAS-20 scores in the present study (before controlling for depression), it could be speculated that different psychotherapeutic mechanisms of change have a different impact on the outcome depending on the patients' level of alexithymia. However, such conclusions have to be drawn with caution, since the sample used to analyze interactions between motivation, alexithymia, and the outcome was more heterogeneous and received a more multimodal/less manualized (psychiatric day hospital) treatment (37) than the outpatient sample of the present study on interactions between the alliance, alexithymia, and the outcome. More research is needed to inform therapists which psychotherapeutic mechanism/s of change [alliance, motivation, resource activation, problem actuation, mastery, clarification, insight, installation of hope, expectations, therapy techniques, ...; see for example, Ref. (38-41)] is/are more or less beneficial for which kind of patients within a diagnostic category.

The result that alexithymia did not moderate-before and after controlling for depression-associations between the patients' alliance ratings and the outcome might be attributable (at least to a certain extent) to the lacking ability of patients with higher alexithymia to adequately rate the relationship with the therapist due to their emotion-processing deficits.

One limitation of the present study is that the therapeutic alliance was assessed only once at the end of the intervention. The therapeutic alliance can fluctuate during psychotherapy and it would be more appropriate to measure the alliance on a session-to-session basis. Another shortcoming of the present study is the solely use of a self-rating to assess alexithymia. Although the TAS-20 is a frequently used alexithymia questionnaire, observer-based ratings might be less biased measures of alexithymia (11). A further limitation is the correlational design with its associated threats to the internal validity. One potential confounder in the context of the present study might be a difference in the therapists' treatment adherence between patients with higher and lower alexithymia, especially regarding the PIT component "establishment of a therapeutic alliance." However, the non-significant correlations between the therapists' adherence ratings and patients' alexithymia for all 12 PIT sessions indicate that treatment adherence was independent from alexithymia. The results that the therapists' as well as the patients' ratings of the therapeutic alliance were not significantly associated with alexithymia - contrary to Quilty et al. (8)—offer further evidence that therapists did not differ in their adherence to the PIT component "establishment of a therapeutic relationship" between patients with higher and lower alexithymia. Nevertheless, there are numerous other potential confounders and the internal validity of the results would have been stronger if patients with higher and also patients with lower alexithymia had been randomized to either a PIT condition including the component "establishment of the therapeutic relationship" or to a PIT condition excluding this component. Yet, it is also questionable whether it is ethical and feasible to conduct such component studies with regard to the therapeutic alliance.

\section{ETHICS STATEMENT}

The multicenter randomized controlled trial ("PISO trial") the data was drawn from was registered (ISRCTN23215121) and approved by the ethics committees of the six participating sites. Informed consent was obtained from all patients. 


\section{AUTHOR CONTRIBUTIONS}

TP drafted and revised the manuscript, contributed to the study design, and performed statistical analyses; HS revised the manuscript, contributed to the study design, and performed statistical analyses; $\mathrm{HG}$ revised the manuscript and contributed to the study design; $\mathrm{PH}$ was the grant recipient of the PISO trial, revised the manuscript, and contributed to the study design; JK revised the manuscript and contributed to the study design; GS revised the manuscript and contributed to the study design; CL drafted and revised the manuscript and contributed to the study design.

\section{REFERENCES}

1. Kleinstäuber M, Witthöft M, Hiller W. Efficacy of short-term psychotherapy for multiple medically unexplained physical symptoms: a meta-analysis. Clin Psychol Rev (2011) 31:146-60. doi:10.1016/j.cpr.2010.09.001

2. Holsting AF, Pedersen HF, Rask MT, Frostholm L, Schröder A. Is psychotherapy for functional somatic syndromes harmful? A mixed methods study on negative effects. J Psychosom Res (2017) 98:113-21. doi:10.1016/j. jpsychores.2017.05.010

3. Zonneveld LN, van Rood YR, Kooiman CG, Timman R, van't Spijker A, Busschbach JJ. Predicting the outcome of a cognitive-behavioral group training for patients with unexplained physical symptoms: a one-year follow-up study. BMC Public Health (2012) 12:848. doi:10.1186/1471-2458-12-848

4. Leibbrand R, Hiller W, Fichter MM. Effect of comorbid anxiety, depressive, and personality disorders on treatment outcome of somatoform disorders. Compr Psychiatry (1999) 40:203-9. doi:10.1016/S0010-440X(99)90004-4

5. Timmer B, Bleichhardt G, Rief W. Importance of psychotherapy motivation in patients with somatization syndrome. Psychother Res (2006) 16:348-56. doi:10.1080/10503300500485292

6. Ogrodniczuk JS, Piper WE, Joyce AS. Effect of alexithymia on the process and outcome of psychotherapy: a programmatic review. Psychiatry Res (2011) 190:43-8. doi:10.1016/j.psychres.2010.04.026

7. Rufer M, Albrecht R, Zaum J, Schnyder U, Mueller-Pfeiffer C, Hand I, et al. Impact of alexithymia on treatment outcome: a naturalistic study of shortterm cognitive-behavioral group therapy for panic disorder. Psychopathology (2010) 43:170-9. doi:10.1159/000288639

8. Quilty LC, Taylor GJ, McBride C, Bagby RM. Relationships among alexithymia, therapeutic alliance, and psychotherapy outcome in major depressive disorder. Psychiatry Res (2017) 254:75-9. doi:10.1016/j.psychres.2017.04.047

9. Bagby RM, Taylor GJ, Parker JDA. The twenty-item Toronto Alexithymia Scale - I. Item selection and cross-validation of the factor structure. J Psychosom Res (1994) 38:23-32. doi:10.1016/0022-3999(94)90005-1

10. Samur D, Tops M, Schlinkert C, Quirin M, Cuijpers P, Koole SL. Four decades of research on alexithymia: moving toward clinical applications. Front Psychol (2013) 4:861. doi:10.3389/fpsyg.2013.00861

11. Waller E, Scheidt CE. Somatoform disorders as disorders of affect regulation: a study comparing the TAS-20 with non-self-report measures of alexithymia. J Psychosom Res (2004) 57:239-47. doi:10.1016/S0022-3999(03)00613-5

12. Karukivi M, Saarijärvi S. Development of alexithymic personality features. World J Psychiatry (2014) 4:91-102. doi:10.5498/wjp.v4.i4.91

13. Mattila AK, Kronholm E, Jula A, Salminen JK, Koivisto AM, Mielonen RL, et al. Alexithymia and somatization in general population. Psychosom Med (2008) 70:716-22. doi:10.1097/PSY.0b013e31816ffc39

14. Saariaho AS, Saariaho TH, Mattila AK, Karukivi MR, Joukamaa MI. Alexithymia and depression in a chronic pain patient sample. Gen Hosp Psychiatry (2013) 35:239-45. doi:10.1016/j.genhosppsych.2012.11.011

15. Schwarz J, Rief W, Radkovsky A, Berking M, Kleinstäuber M. Negative affect as mediator between emotion regulation and medically unexplained symptoms. J Psychosom Res (2017) 101:114-21. doi:10.1016/j.jpsychores.2017.08.010

16. Rief W, Heuser J, Fichter MM. What does the Toronto Alexithymia Scale TAS-R measure? JClin Psychol (1996) 52:423-9. doi:10.1002/ (SICI)1097-4679(199607)52:4<423::AID-JCLP6>3.0.CO;2-Q

\section{ACKNOWLEDGMENTS}

This work was supported by the German Research Foundation (DFG) and the Georg-August-University Göttingen (Germany) within the funding programme Open Access Publishing.

\section{FUNDING}

The German Research Foundation (DFG; He 3200/4-1) funded this study.

17. Rasting M, Brosig B, Beutel ME. Alexithymic characteristics and patienttherapist interaction: a video analysis of facial affect display. Psychopathology (2005) 38:105-11. doi:10.1159/000085772

18. Ogrodniczuk JS, Piper WE, Joyce AS. The negative effect of alexithymia on the outcome of group therapy for complicated grief: what role might the therapist play? Compr Psychiatry (2005) 46:206-13. doi:10.1016/j.comppsych.2004.08.005

19. Horvath AO, Del Re AC, Flückiger C, Symonds D. Alliance in individual psychotherapy. Psychotherapy (2011) 48:9-16. doi:10.1037/a0022186

20. Lorenzo-Luaces L, DeRubeis RJ, Webb CA. Client characteristics as moderators of the relation between the therapeutic alliance and outcome in cognitive therapy for depression. J Consult Clin Psychol (2014) 82:368-73. doi:10.1037/ a0035994

21. Zilcha-Mano S, Errázuriz P. One size does not fit all: examining heterogeneity and identifying moderators of the alliance-outcome association. J Couns Psychol (2015) 62:579-579. doi:10.1037/cou0000103

22. Sattel H, Lahmann C, Gündel H, Guthrie E, Kruse J, Noll-Hussong M, et al Brief psychodynamic interpersonal psychotherapy for patients with multisomatoform disorder: randomised controlled trial. Br J Psychiatry (2012) 200:60-7. doi:10.1192/bjp.bp.111.093526

23. Kroenke K, Spitzer RL, deGruy FV, Hahn SR, Linzer M, Williams JB, et al. Multisomatoform disorder: an alternative to undifferentiated somatoform disorder for the somatizing patient in primary care. Arch Gen Psychiatry (1997) 54:352-8. doi:10.1001/archpsyc.1997.01830160080011

24. Hintikka J, Honkalampi K, Lehtonen J, Viinamäki H. Are alexithymia and depression distinct or overlapping constructs? A study in a general population. Compr Psychiatry (2001) 42:234-9. doi:10.1053/comp.2001.23147

25. Parker JD, Bagby RM, Taylor GJ. Alexithymia and depression: distinct or overlapping constructs? Compr Psychiatry (1991) 32:387-94. doi:10.1016/0010-440X(91)90015-5

26. First MB, Spitzer RL, Gibbon M, Williams JBW. Structured Clinical Interview for DSM-IV-TR Axis I Disorders. New York: Biometrics Research, New York State Psychiatric Institute (2002).

27. Arbeitskreis PISO. Somatoform Disorders. Psychodynamic-Interpersonal Therapy (PISO) [in German]. Göttingen: Hogrefe (2012).

28. Chernyak N, Sattel H, Scheer M, Baechle C, Kruse J, Henningsen P, et al. Economic evaluation of brief psychodynamic interpersonal therapy in patients with multisomatoform disorder. PLoS One (2014) 9:e83894. doi:10.1371/ journal.pone.0083894

29. Probst T, Sattel H, Henningsen P, Gündel H, Lahmann C. Alexithymia as a moderator of treatment outcomes in a randomized controlled trial on brief psychodynamic interpersonal psychotherapy for patients with multisomatoform disorder. Psychother Psychosom (2017) 86:57-9. doi:10.1159/ 000448287

30. Haack MBF. The Adherence of Psychotherapists in a Newly Developed Manualized Brief Therapy: Association with Patient Characteristics and Therapeutic Outcome [in German] [Dissertation]. Munich: Technische Universität München (2010).

31. Ware JE, Sherbourne CD. The MOS 36-item Short-Form Health Survey (SF-36): I. Conceptual framework and item selection. Med Care (1992) 30:473-83. doi:10.1097/00005650-199206000-00002

32. Luborsky L. Principles of Psychoanalytic Psychotherapy. A Manual for Supportive-Expressive Psychotherapy. New York: Basic Books (1984). 
33. Kroenke K, Spitzer RL, Williams JB. The PHQ-9: validity of a brief depression severity measure. JGen Intern Med (2001) 16:606-13. doi:10.1046/j.1525-1497.2001.016009606.x

34. Hayes AF. Introduction to Mediation, Moderation, and Conditional Process Analysis: A Regression-Based Approach. New York: Guilford Press (2013).

35. Taylor GJ, Bagby RM, Parker JDA. Disorders of Affect Regulation: Alexithymia in Medical and Psychiatric Illness. Cambridge: Cambridge University Press (1997).

36. Li S, Zhang B, Guo Y, Zhang J. The association between alexithymia as assessed by the 20 -item Toronto Alexithymia Scale and depression: a meta-analysis. Psychiatry Res (2015) 30:1-9. doi:10.1016/j.psychres.2015.02.006

37. Terock J, Janowitz D, Grabe HJ, Freyberger HJ, Schneider W, Klauer T. Alexithymia and psychotherapeutic treatment motivation: main and interactional effects on treatment outcome. Psychother Psychosom (2017) 86:185-6. doi:10.1159/000457961

38. Mander JV, Wittorf A, Schlarb A, Hautzinger M, Zipfel S, Sammet I. Change mechanisms in psychotherapy: multiperspective assessment and relation to outcome. Psychother Res (2013) 23:105-16. doi:10.1080/10503307.2012.744111

39. Mander JV, Schlarb A, Teufel M, Keller F, Hautzinger M, Zipfel S, et al. The individual therapy process questionnaire (ITPQ): development and validation of a revised measure to evaluate general change mechanisms in psychotherapy. Clin Psychol Psychother (2015) 22:328-45. doi:10.1002/cpp.1892

40. Tschacher W, Junghan UM, Pfammatter M. Towards a taxonomy of common factors in psychotherapy-results of an expert survey. Clin Psychol Psychother (2014) 21:82-96. doi:10.1002/cpp.1822

41. Wampold BE. How important are the common factors in psychotherapy? An update. World Psychiatry (2015) 14:270-7. doi:10.1002/wps.20238

Conflict of Interest Statement: The authors declare that the research was conducted in the absence of any commercial or financial relationships that could be construed as a potential conflict of interest.

Copyright @ 2017 Probst, Sattel, Gündel, Henningsen, Kruse, Schneider and Lahmann. This is an open-access article distributed under the terms of the Creative Commons Attribution License (CC BY). The use, distribution or reproduction in other forums is permitted, provided the original author(s) or licensor are credited and that the original publication in this journal is cited, in accordance with accepted academic practice. No use, distribution or reproduction is permitted which does not comply with these terms. 